Dennis S.C. Lam ${ }^{1}$

Ann P. Koehler ${ }^{2}$

Dorothy S.P. Fan ${ }^{1}$

Wah Cheuk'

Alfred T.S. Leung ${ }^{1}$

Joan S.K. $\mathrm{Ng}^{1}$

${ }^{1}$ Department of Ophthalmology and Visual Sciences and

${ }^{2}$ Department of Microbiology

The Chinese University of Hong Kong

Prince of Wales Hospital

Shatin, N.T., Hong Kong

Dr Dennis S.C. Lam, FRCS, FRCOphth

Eye Unit, Prince of Wales Hospital

Department of Ophthalmology and Visual Sciences

The Chinese University of Hong Kong

Shatin, N.T., Hong Kong

Tel: (852) 26322881

Fax: (852) 26482943

e-mail: dennislam@cuhk.edu.hk

Sir,

\section{Endogenous endophthalmitis due to group G streptococcus}

Group G streptococcus is a rare cause of endogenous endophthalmitis. ${ }^{1-4}$ While intravitreal antibiotics have an established role in the treatment of endogenous endophthalmitis, ${ }^{4}$ the value of immediate vitrectomy is uncertain. A case of bilateral endogenous endophthalmitis due to group G streptococcus is reported in which only one eye was treated with immediate vitrectomy.

\section{Case report}

A 69-year-old man presented with infective endocarditis due to group $G$ streptococcus following a 2 day history of general malaise and pyrexia. Blood cultures on admission, using the BACTEALERT system, yielded large colony type group $\mathrm{G}$ beta-haemolytic streptococcus that was sensitive to penicillin. The patient received intravenous benzylpenicillin ( $1.2 \mathrm{~g} 4$ hourly) and intravenous gentamicin ( $80 \mathrm{mg}$, twice daily) for their synergistic effect.

Five days following admission, while the patient was on intravenous antibiotics, he complained of rapid deterioration in vision in both eyes. His visual acuity was hand movements in each eye. There was no relative afferent pupillary defect. Bilateral anterior uveitis (without hypopyon) was present. While the right pupil was successfully dilated, it was not possible to dilate the left pupil due to extensive posterior synechiae. Both eyes had a dense vitritis which prevented fundus visualisation, but no retinal pathology was apparent on ultrasonography.

Bilateral endogenous endophthalmitis was diagnosed. Intravitreal vancomycin (1.0 mg), gentamicin $(0.1 \mathrm{mg})$ and dexamethasone $(0.4 \mathrm{mg})$ were administered to both eyes. This combination was chosen because of their synergistic effect against group G streptococcus. ${ }^{5}$ The right eye also underwent an immediate pars plana vitrectomy. At surgery, a subretinal abscess was found in the inferotemporal periphery with necrotic overlying retina. An iatrogenic retinal tear occurred at this site that was treated with cryotherapy and a scleral buckle. Vitreous samples were inoculated onto enrichment media (with beta-lactamase), blood agar and heated blood agar. No organisms were isolated on incubation for 5 days. An immediate vitrectomy was not performed in the left eye because of poor pupil dilatation. Postoperatively, both eyes were treated with dexamethasone $0.1 \%$ ( 2 hourly) and atropine $1 \%$ (twice daily). The patient also continued to receive intravenous benzylpenicillin and gentamicin for 6 weeks as treatment for endocarditis.

At 1 week following surgery, a retinal detachment developed in the right eye due to the iatrogenic retinal tear. This was treated with vitrectomy, endolaser retinopexy and internal tamponade with $14 \%$ sulphur hexafluoride $\left(\mathrm{SF}_{6}\right)$. Surgery was combined with a phacoemulsification cataract extraction to improve visualisation since a significant cataract had developed in this eye. The retina remained flat following surgery. A secondary posterior chamber intraocular lens implant was performed 3 months later that resulted in a best corrected visual acuity of $6 / 7.5$.

The left eye improved rapidly following intravitreal antibiotics and steroid. Therefore, this eye did not undergo a vitrectomy. As the vitritis cleared, no retinal abscess was observed. The best corrected visual acuity in this eye was $6 / 9$, with a clear vitreous, at 18 months following the onset of endophthalmitis.

\section{Comment}

Beta-haemolytic streptococci are classified according to their Lancefield group. ${ }^{6}$ Group A organisms are the predominant pathogenic streptococci in man, while those of other groups (B, C and $\mathrm{G}$ ) are occasionally involved. Group $G$ streptococci may occur as normal commensals in the nasopharynx, skin, intestine and vagina. They are a rare cause of sinusitis, pharyngitis, pulmonary infections, septic arthritis and endocarditis. ${ }^{6}$

Five cases of endogenous endophthalmitis due to group G streptococcus have been reported. ${ }^{1-4}$ These cases were associated with endocarditis (3 cases), cellulitis of the foot ( 1 case) and facial trauma ( 1 case). Both eyes were affected in 2 cases. The visual outcome was uniformly poor, but 3 of these patients were treated only with systemic antibiotics ${ }^{1-3}$ and another received only systemic and subconjunctival antibiotics. ${ }^{4}$

Vitrectomy with intravitreal antibiotics was performed in 1 case, but the interval between the onset of endophthalmitis and this treatment was not recorded. ${ }^{2}$ The benefit of intravitreal, in addition to systemic, antibiotics for endogenous endophthalmitis is clearly established. ${ }^{7}$ Intravitreal steroid administration may also be beneficial by reducing intraocular inflammation. ${ }^{8}$ Our patient demonstrates that a good visual outcome can be 
achieved in group $\mathrm{G}$ streptococcal endophthalmitis with prompt administration of intravitreal antibiotics and steroid.

The value of immediate vitrectomy in the treatment of endogenous endophthalmitis is uncertain. Theoretical advantages include rapid removal of the infecting organisms and toxins, clearing of vitreous opacities, collection of a large vitreous sample for culture and possibly better distribution of intravitreal antibiotics. Vitrectomy may, however, be technically difficult in such cases and is associated with complications such as cataract and retinal detachment. ${ }^{9}$

Immediate vitrectomy was considered in our patient because of rapidly progressive cellular infiltration of the vitreous. While the severity of endophthalmitis in each eye was similar, immediate vitrectomy was performed in only one eye because of inadequate pupil dilatation in the other eye. Ultimately, both eyes achieved a good visual outcome. The eye that underwent an immediate vitrectomy did, however, suffer operative complications and required subsequent surgery for retinal detachment and cataract.

The Endophthalmitis Vitrectomy Study has helped to clarify the role of immediate vitrectomy in acute endophthalmitis after cataract surgery. The findings of this study cannot, however, be extrapolated to the management of endogenous endophthalmitis.

Appropriate treatment of this heterogeneous disorder depends on both the causative organism and the clinical setting. For the treatment of group G streptococcal endogenous endophthalmitis, however, this case report suggests that immediate vitrectomy with intravitreal antibiotics offers no advantage compared with the administration of intravitreal antibiotics alone.

\section{References}

1. Berkey P, Rolston K. Group G streptococci as a cause of bacterial endophthalmitis. Arch Ophthalmol 1988;106:171-2.

2. Verweij P, Rademakers A, Koopmans P, Meis J. Endophthalmitis as a presenting symptom of Group G streptococcal endocarditis. Infection 1994;22:56-7.

3. Puliafito C, Baker A, Haaf J, Foster C. Infectious endophthalmitis: a review of 36 cases. Ophthalmology 1982;89:921-9.

4. Farber BP, Weinbaum DL, Dummer SJ. Metastatic bacterial endophthalmitis. Arch Intern Med 1985;145:62-6.

5. Gaunt PN, Seal DV. Group G streptococcal infections. J Infect 1987;15:5-20.

6. Shanson D. Microbiology in clinical practice. 2nd ed. Bristol: Wright, 1989.

7. Forster R, Zachary I, Cottingham A, Norton E. Further observations on the diagnosis, cause and treatment of endophthalmitis. Am J Ophthalmol 1976;81:52-6.

8. Park S, Samity N, Ruoff K, D'Amico, Baker A. Effect of intravitreal dexamethasone in treatment of pneumococcal endophthalmitis in rabbits. Arch Ophthalmol 1995;113:1324-9.

9. The Endophthalmitis Vitrectomy Study Group. Results of the Endophthalmitis Vitrectomy Study: a randomised trial of immediate vitrectomy and of intravenous antibiotics for the treatment of postoperative bacterial endophthalmitis. Arch Ophthalmol 1995;113:1479-96.
Jennifer H.Y. Tan

Douglas K. Newman

Robert L. Burton

Department of Ophthalmology

West Norwich Hospital

Bowthorpe Road

Norwich NR2 3TU, UK

Dr Jennifer Tan

Department of Ophthalmology

St James's University Hospital

Leeds LS9 7TF, UK

Tel: +44 (0)1132064602

Fax: +44 (0)1132065028

e-mail: eyes@jenni.globalnet.co.uk

\section{Sir,}

\section{Combined limbal and corneal autograft transplantation}

The technique of limbal autograft transplantation has been well described by several authors in the acute and chronic management of alkali-injured eyes. ${ }^{1-4}$ Studies have shown that autograft transplantation of uninjured limbal tissue results in a fast regeneration of normal corneal epithelium with improvement of symptoms. This technique also promotes regression of corneal neovascularisation with a subsequent improvement in the prognosis for a future penetrating keratoplasty. ${ }^{4,5}$ Our case is unique in that all the surgical rehabilitation was performed at one operation, with all the tissue coming from the patient himself as a result of a series of unfortunate events.

\section{Case report}

A 57-year-old man presented with a deterioration in his right visual acuity due to a serous retinal detachment with an underlying choroidal malignant melanoma. Unfortunately, the right eye had been the better seeing eye. In 1979 he had sustained a severe corneal alkali injury to this left eye, with a reduction of vision in this eye to 6/60 (Fig. 1). He was referred to an ophthalmic oncologist for an opinion regarding management of his right choroidal melanoma. Due to the large size of the tumour it was not technically possible to salvage the right eye by more conservative treatment. After

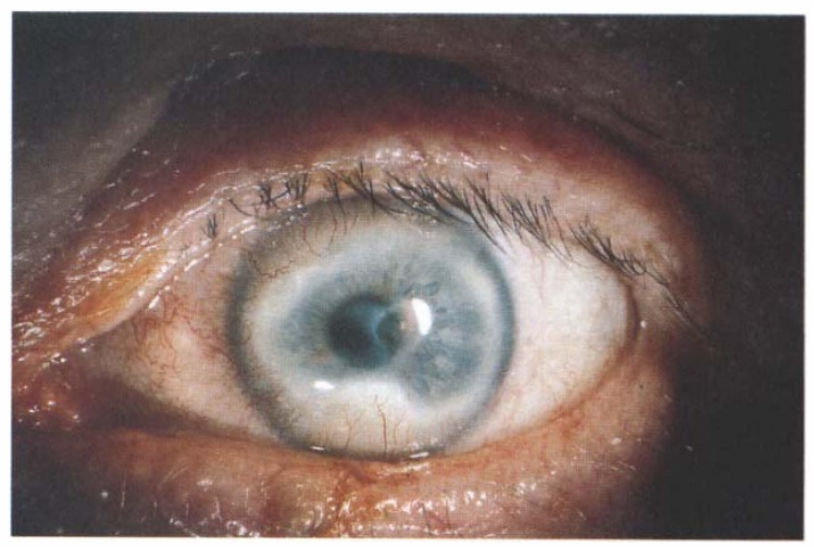

Fig. 1. Left eye with extensive corneal scarring resulting from alkali injury. 